\title{
Low Energy Clustering Adaptation Protocol for an Adhoc Wireless Sensor Network
}

\author{
N. M. Murad \\ LE²P laboratory, University of Reunion \\ 15, avenue René Cassin \\ 97400 Saint Denis - France
}

\begin{abstract}
Energy dissipation in a Wireless Sensor Network is an active research field. Energy dissipation is related to the network topology and protocol used to route data. So, two criteria are proposed to elect a relay. A linear or a random choice from a set of potential nodes to become a relay based on their energy level above a mean threshold energy. These criteria improve the organization of the node communications and the lifetime of the network. The criteria and the protocol called the Self Organization One Hop Low Energy Clustering Adaptation (SOORLECA) are explained. The SOORLECA is designed for environmental and military monitoring with precise requisite measures at each node. No mean data or smoothing data technique is required at the Sink Node (SN). Random topology of the network for 100 nodes over $100 \mathrm{~m}^{2}$ are simulated to show the protocol performances compared to direct transmission of the node to the $\mathrm{SN}$, short routing path and static clustering protocols. The lifetime and the dissipated energy of the network are our criteria to measure the performance of the WSN. It is shown that the SOORLECA ACMET1 improves $2 x$ the lifetime of network with $61 \%$ dissipated energy after $50 \%$ of nodes have dead.
\end{abstract}

Keywords- Wireless sensor network, low energy consumption, lifetime network, one hop relay, clustering, balanced energy dissipation.

\section{INTRODUCTION}

Wireless sensor networks (WSN) have been a very active research field in recent years. WSN have numerous advantages. They are easier, faster and cheaper to deploy than wired networks. They have a large coverage area and longer range. They have a higher degree of fault tolerance than other wireless networks. Failure of one or a few nodes affects the monitoring operation of the network. Finally, they are self-configuring and self-organizing. These advantages make them very promising in a wide range of applications, especially in environment and military monitoring. For our case, precise measures are required at each instant.

Many routing protocols have been proposed for sensor networks to make these networks practical and efficient, such as directed diffusion [1], LEACH [2], and two-tier data dissemination (TTDD) [3]. These protocols attempt to make the constituents nodes work in unison to achieve specific tasks. Invariably they seek to minimize energy spending and maximize the network lifetime. Some use hierarchical design in their communication model [2],[5],[6],[7],[13] some such as SPIN and directed diffusion use planar multi-hop design with various optimization techniques such data aggregation and minimal data dissemination [4],[11],[12].
Moreover, energy efficient data dissemination is among the first set of research issues being addressed. The SPIN is one of the early works that focuses on efficient dissemination of an individual sensor's observation to all the sensors in a network [4]. However, most routing protocols did not consider the uneven energy consumption problem in sensor networks. In typical sensor networks, the many-to-one traffic pattern is dominant, that is, a large number of sensor nodes send data to the one or a few SN. Sensor nodes near the SN need to relay packets for all other sensors and have much heavier traffic burden. These sensors run out of power much faster than other nodes, and they are referred to as critical nodes. The short lifetime of the critical nodes dramatically reduces the sensor network lifetime. The SOORLECA could be a solution for this kind of problem.

Also, the main goal of this paper is to maximize the lifetime of the WSN and to minimize the expenditure of the energy across the network. The extension of this algorithm with a second criterion is used to balance the life of the nodes in each cluster. But the lifetime of the WSN is reduced compared to the first version of the protocol [14].

Here, the data transmission deadline is not taken into account in the network. Interferences between nodes in the same cluster and interferences between nodes with other clusters are minimized thanks to the TDMA access and the power control at each node. The CSMA MAC and TDMA PHY are used as access protocols in our WSN. There are some constraints about delay processing and delay propagation in the network. But, as the data rate is $2 \mathrm{Mbps}$ and the surface $\mathrm{S}$ is small, we assume that these points are quite negligible. Any CDMA radio interface could be used. But, the node becomes expensive and the energy consumption is significant, instead the CDMA interface reduces the radio interference.

In this work, we consider a WSN where:

- A one hop transmission is considered. The node sends messages to the sink node ( $\mathrm{SN}$ ) via an only one chosen relay.

- The sink node is fixed and is among the nodes on the playing field $\mathrm{S}$,

- A heterogeneous nodes randomly positioned is grouped in cluster. The size of the cluster is a significant factor that we will see later. Intuitively, it is necessary that the clusters of nodes should be 
homogeneous. But in real application, the position of the nodes is random.

- We assume that the position of the nodes is known thanks to a GPS or with another algorithm across the network that gives the relative position of the nodes. It is assumed that the energy spent by this algorithm is known and is constant. Because, as the nodes are fixed, it is sufficient to know the position of the nodes once and for all.

- The lifetime of the network is significant. And hence, if any nodes are dead, the network is considered dead. Indeed, the local optimization in each cluster of the energy dissipation allows increasing the lifetime of the network.

- The SN wants exactly the data measured at each node. It means no averaging or smoothing technique at the relay layer is made. Because, when data is aggregated at the relay layer, the size of the message increases. Accordingly, the time to send the data and the dissipated energy increases. In our work, the size of the message is constant. An optimization is done, by using scrambling technique to keep the size of the message constant when the messages are sent across the network.

This paper focuses on the improvement of the SOORLECA proposed protocol in [14] with balance lifetime performance increase. The details of the communication operations, the data merging at the relay, the scrambling of data measured at node and the criterion decision to choose a relay are explained in section II. Then, a first order radio model and the energy computation are shown in section III. Section IV discusses on the performances and on the operations of the direct transmission, of the minimum transmission and routing energy, of the static cluster, of the LEACH algorithms compared to the SOORLECA one. To finish, a conclusion and some future works are discussed.

\section{SOORLECA ALGORITHM}

The main characteristic of the SOORLECA algorithm is detailed in this section. We have improved the development of the SOORLECA [14] in adding a second criterion ACMET2 that balances the life of nodes in each cluster.

A related work as LEACH [2] works following the mean or smooth of data at the relay, the choice of a number of relays following a probability rule over the network and therefore a variable size of cluster at each communication round.

SOORLECA algorithm is different because five relays are chosen randomly and the algorithm builds five groups of nodes around the five relays. Hence, the number of nodes in each cluster is fixed during the life of the network except if a node is dead. On the other hand, each node in a given cluster becomes by turns a relay following a criterion based on energy level (section C). Also, at the relay layer, all received data of measures from nodes are merged intelligently in using a scrambling sequence. Finally, the SN knows exactly the measures from each node.
A cycle of communication is defined as the quantum time simulation parameter to purchase a measure, to format it in a frame, to send it to the following node and to receive it and to process it.

Section A describes the format of the frame message. The assumptions and the operations at the nodes, at the relay node and at the sink node are explained in the sections B, C and D. To finish, the SOORLECA algorithm is detailed with its initial phase (section E) and its current functioning phase (section F).

\section{A. The frame of messages}

The $k$-bits frame message of 2000 bits is composed by a header and a payload. The length of the header and the payload fields are 464 bits and 1536 bits, respectively. Let us take a particular application like environment monitoring on temperature measurement for understanding. The data of temperature is coded over 12 bits with an Analogic to Digital Converter. Let us note this measure $b_{k}$ as the $k$-th node data measured. Then a scrambling sequence $h_{k}$ of size $L$ times $b_{k}$ gives the final size of the payload, noted $P_{k}$. $P_{k}$ will be the payload of the $k$-th node. Here, $L=128$. $L$ would be greater than or equal of the number of node $N(L \geq N)$. The scrambling operation is done by each node with its own scrambling sequence. The idea about the scrambling sequence is explained later (see section $\mathrm{C}$ ).

\section{B. The assumptions on the nodes}

Assumptions concerning nodes are made below. The total number of nodes $N$ is known and is dispersed randomly on a surface $S$. $N$ will be a power of two. An equal energy is distributed initially for each node $\left(E_{\text {node }}=0.5 \mathrm{~J} /\right.$ node $)$. All nodes have an omnidirectional antenna. Each node $k$ is numbered from 1 to $N$. The index of the node is the name of the node and the name does not change during the life of the WSN. Each $k$-th node is associated with the $k$-th scrambling sequence $h_{k}$. The scrambling code is built from Hamming sequence [13]. The $k$-th number of Hamming sequence $h_{k}$ related to the $k$-th node takes easy the sink node to get the desired data measured from the desired node $k$.

From the radio point of view, the transmission power of each node is supposed to reach all nodes on S. A power control exists in each node. All nodes are not mobile and then the channel propagation variation is quasi-null. But it could have some slow apparent movement of object through the playing field $\mathrm{S}$. The channel transmission is modeled as a path loss channel model (section III). The energy to receive and to process the data is constant and is noted $E_{r x}$.

\section{The relay operations}

From [2], the optimal choice of relay is five for 100 nodes. Five clusters will be built up. Here, node transmission is done with one hop to the SN. So, node information is sent through the relay. A cluster is a group of nodes bound to the relay. The size of the cluster does not change during the life of the WSN except when the node is dead. In a cluster, the re-election of the relay among the nodes is done following the Alive node in Cluster with a Mean Energy Threshold criterion called 
ACMET. A priori, if the same relay is solicited, its lifetime goes down quickly (section IV.C). Thus, each node within the cluster will become a relay. So, the election relay for the next round is done following the ACMET criterion.

1) The Alive node in a Cluster with a Mean Energy Threshold (ACMET) criterion

Each time that the nodes send data to the relay, they send also their energy level in the header field. Then, the relay records the set of the energy levels and chooses the coming node which will be the future relay following two sub criterions:

ACMET 1: The node getting the greatest energy level among the entire nodes in the cluster is chosen as the future relay.

ACMET 2: A random node is chosen as the future relay among the entire nodes which their energy levels is greater than the mean of all energy levels of nodes in the current cluster.

The relay inserts in the header field the name of the future relay and sends the final message noted $\mathrm{P}$ to the $\mathrm{SN}$ (see step 7 in section E and step 5' in section F). At the same time, all the nodes are in listen passive mode. Now, they know in the next round who is the next relay and in which order they will send their data.

2) The data processing and the fusion data at the relay node

Let us consider there are $\Theta$ nodes in a certain cluster. So at each node $k$, the data $b_{k}$ is spread by $h_{k}$ and gives $P_{k} . P_{k}$ is sent to the relay. The relay will receive $\Theta$ messages of $P_{k}$. It adds all $P_{k}$ payloads (figure 1). It is our fusion data technique. Finally, only one new payload $\mathrm{P}$ from the relay is sent to the $\mathrm{SN}$ without increasing the message size.

As an example, figure 1 explains simply the mechanism without taking into account the attenuation of the transmission channel, the errors related to the bit synchronization, the modulation, the demodulation and the decoding technical decision.

Let us take a Hamming matrix $H$ of size $4 \times 4$. We extract four sequences of size four, noted $h_{1}$ to $h_{4}$ in (1). Hamming sequence was chosen because it has particular auto correlation and inter correlation statistical properties [9],[13].

$$
H=\left(\begin{array}{l}
h_{1} \\
h_{2} \\
h_{3} \\
h_{4}
\end{array}\right)=\left(\begin{array}{cccc}
1 & 1 & 1 & 1 \\
1 & -1 & 1 & -1 \\
1 & 1 & -1 & -1 \\
1 & -1 & -1 & 1
\end{array}\right)
$$

Four nodes are considered. The size of $b_{k}$ and $P_{k}$ is 3 bits and 4 bits, respectively. One of the four nodes is a relay. We see in figure 1 that the SN could retrieve the initial message $b_{3}$ of node 3 .

\section{The sink node operations}

In this section, we explain how the SN works, how measures are collected and how the SN knows passively which node number is in each cluster.

By default the SN knows exactly the number of nodes $N$ over $S$ at the beginning of the life WSN. After receiving five messages from the five relays (step 2), the SN knows the name of each relay. Also, the five $P$ messages received at the $\mathrm{SN}$ (step 7) allow a confirmation and an update of the measures database and the state node database. In the step 7, the SN knows the node name within each cluster. Thus, the five conceived clusters do not vary, except when the node relay within the cluster changes and if a node is dead. The size of the cluster does not vary, means that no nodes change cluster during the life of the network.

Finally, the SN knows exactly and passively the state of the WSN. During the life of the network, the SN knows if a node is dead or alive. Because, when the SN descrambles the message with the sequence $H_{k}$ for the $k$-th node (figure 1) and if the result is quite null, then SN concludes after a certain time that the node is dead.

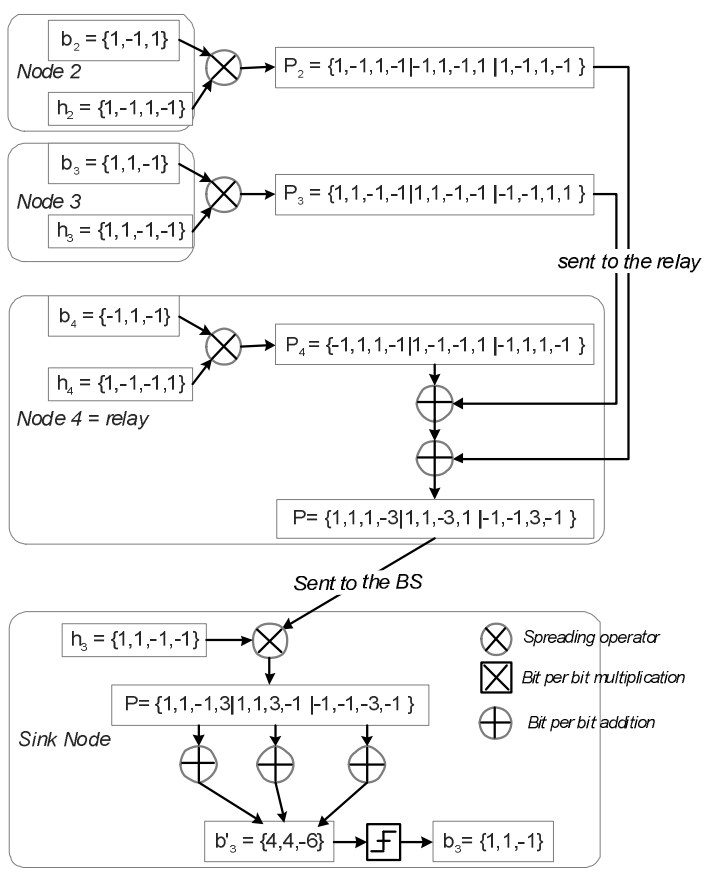

Figure 1. Fusion data with a scrambling operation.

\section{E. The ininial phase: Operation mechanism}

As a preamble, the $\mathrm{SN}$ is in or outside the playing field $\mathrm{S}$. Each data from each node must be transmitted without alteration or compression at the relay level. Our algorithm strategy is different from [2]. The SN will know precisely the data coming from each node.

At the beginning of the life of the network, all nodes are in receipt mode and wait first for the SN message pilot. 
Step 1 - The SN sends to all nodes a broadcast message to ask for an election of five relays among $N$. SN indicates also the length response window noted $W$.

Step 2 - Election of the five relays following the ACMET criterion. The five relays broadcast a message notifying their names (number of nodes). The sending of the five broadcast messages is done randomly within the $W$ window.

Step 3 - The decisions of the nodes and the SN action.

Step 3.a - Following the strength of the reception power of the five received messages, the node decides which relay it is going to link. Knowing the strength reception power, the node uses power control in order to reduce nodes interferences for future communication. Thus, all nodes shape a cluster around the relay. Finally, there are five created clusters.

Step 3.b - In the same way, the SN obtains the name of the five relays.

Step 4 - Each node nearest a relay informs this relay by a message. The message indicates the name of the node which it binds with the relay by using a CSMA MAC protocol.

Step 5 - During a period of time, the relay counts the number of nodes, noted $\Theta$. A period $T$ is divided in $\Theta+1$ equal parts. It means that each node will send cyclically all $T /(\Theta+1)$ periods. The relay sends a message that indicates which instant each node of the cluster can send data message to the actual relay following a TDMA access.

Step 6 - Then all nodes send their data to the relay. During the $(\Theta-1) \cdot T /(\Theta+1)$ length, the node goes to sleep mode to save battery energy.

Step 7 - The relay adds all the payload of the nodes, forms a final message noted $P$ and sends it to the $\mathrm{SN}$ almost the end of the period $T$.

We assume that the steps 1 to 5 are done one or several times. Hence, the energy spent is quantifiable and negligible regarding the rate of data transfer in the network.

\section{F. The routine phase: Local cluster reelection relay}

The clusters of nodes were created following the initial phase (section E, step 3.a). So, each node of a cluster knows the number of nodes in this cluster and at which instant they have to send their own message (step 5). During the cycle, the nodes send to the actual relay their energy level in the header field and their measures in the payload field. The relay merges the received data (section $\mathrm{C}$-2) and chooses the future relay for the next round thanks to the ACMET criterion (section $\mathrm{C}-1$ ). So the steps 4 to 7 of initial phase become as below:

Step 4' - Each node in the cluster send to the actual relay their messages (energy level + data measured) at each instant $(\Theta-1) \cdot T /(\Theta+1)$ (old steps 4 to 6$)$. Then, the node goes to sleep mode or listen passive mode to save battery energy.

Step 5' - During a period of time, the relay counts the number of nodes, noted $\Theta$. It could be that some nodes are dead in the cluster during the life of the WSN and the value of $\Theta$ decreases. A new period $T$ is divided in $\Theta+1$ equal parts.
It means that each node will send cyclically all $T /(\Theta+1)$ in the next cycle. Finally, the relay sends to the SN a message $P$ containing three information essentials (old step 7):

- The measures from all nodes in the cluster which are merged in the final message $P$,

- The name of the future relay for the next cycle,

- At which instant each node of the cluster can send its own message to the future relay following a TDMA access,

As each node is in listen mode, they receive passively the message $P$ for the next cycle of communication.

\section{A FIRST ORDER RADIO MODEL AND ENERGY COMPUTATION}

The same radio model discussed in [2] is applied. To run the node communication in radio high frequency and base band processing, the electronic transmitter (or the receiver) circuitry and the electronic of the digital signal processing dissipate $\mathrm{E}_{\text {elec }}=50 \mathrm{~nJ} / \mathrm{bit}$. The transmitter amplifier dissipates $\mathrm{E}_{\mathrm{amp}}=100 \mathrm{pJ} / \mathrm{bit} / \mathrm{m}^{2}$. The nodes have power control and can expend the minimum required energy to reach the intended following node or sink node. The node can be turned to sleep mode in order to economize battery.

The transmitting and receiving energy are related to the $k$ bits message and the $d$ distance between two nodes.

$$
\left\{\begin{array}{l}
E_{T x}(k, d)=k \cdot E_{\text {elec }}+k \cdot E_{a m p} \cdot d^{2} \\
E_{R x}(k)=k \cdot E_{\text {elec }}
\end{array}\right.
$$

A $d^{2}$ energy loss is used due to the path loss channel model [8],[9]. The radio channel is assumed symmetric between two nodes. So, the energy dissipated to transmit messages from the node $A$ to the node $B$ is equal in the opposite way. A data packet of length $k$-bits $=2000$ bits is transmitted. When $d^{2}=500 \mathrm{~m}^{2}$, note that the energy dissipated in the amplifier RF circuitry equals the energy dissipated in the base band circuitry and consequently, the cost to transmit a message will be twice the cost to receive it.

\section{SimUlations AND ANALYSES}

All curves of performance measure are averaging because a hundred of different topologies of network are simulated. It means that the $N$ nodes do not have the same position at each beginning of the simulation. But they have same position during the simulation. The percentage of live nodes (lifetime of the WSN) and the dissipated energy in the network will be our main criterion to comment the performance of the algorithms.

We remind here that a cycle is the quantum time simulation parameter to purchase a measure, to format it in a frame, to send it to the following node and to receive it and to process it. An event is a measure. And so if an event appears, the measure is processed and sent instantaneously. At the beginning of the simulation, $50 \mathrm{~J}$ is distributed equitably over the $N$ nodes. All subsections below describe succinctly the mechanism of the 
algorithms. Then some comments are done over the obtained curves of results.

\section{A. The Direct Transmission protocol}

In this protocol, all nodes send directly their messages to the sink node. The events (like measures) occur cyclically and arranged at the node. It means that the nodes send directly to the SN following a cyclic sequence $\{1,2, \ldots, N, 1,2, \ldots\}$, called cyclic event. Another manner to transmit directly is that the events occur in following a uniform distribution in $[1, N]$, called uniform event.

When events appear uniformely, the lifetime of the network and the dissipated energy are greater than those of the nodes with the cyclic events (figures 2 and 3 ). Let note that for a cyclic transmission of data, the lifetime of the network is constant during the first 5000 cycles. Then, the performance decreases quickly compared to the lifetime of network with a uniform distribution transmission data.

\section{B. The Minimum Transmission and Routing Energy (MTRE)}

The MTRE consists in searching the relay node nearest from the sink node with one hop transmission. Then, all nodes send their messages to the same relay until it is dead. So, another relay nearest the $\mathrm{SN}$ is elected to be the new relay, and so on, until to find a possible relay node eligible in a threshold distance $d_{t}$ from the SN. As in the previous section, the events occur cyclically or uniformly.

The energy dissipated in the network with MTRE is greater than the direct transmission (figure 2). Figure 3 shows that the lifetime network performance with the MTRE algorithm is poor compared to the direct transmission. It is obvious. Because instead to get only one link transmission, we have two links communications. Also, the energy is much used to reprocess the data of each node at the relay layer. This work is made for each node which sends its messages to the SN. So the relay with MTRE works hard compare to the SOORLECA algorithm where, the relay waits to receive all data from nodes, merges data and sends the final message $\mathrm{P}$ to the $\mathrm{SN}$ only one times.

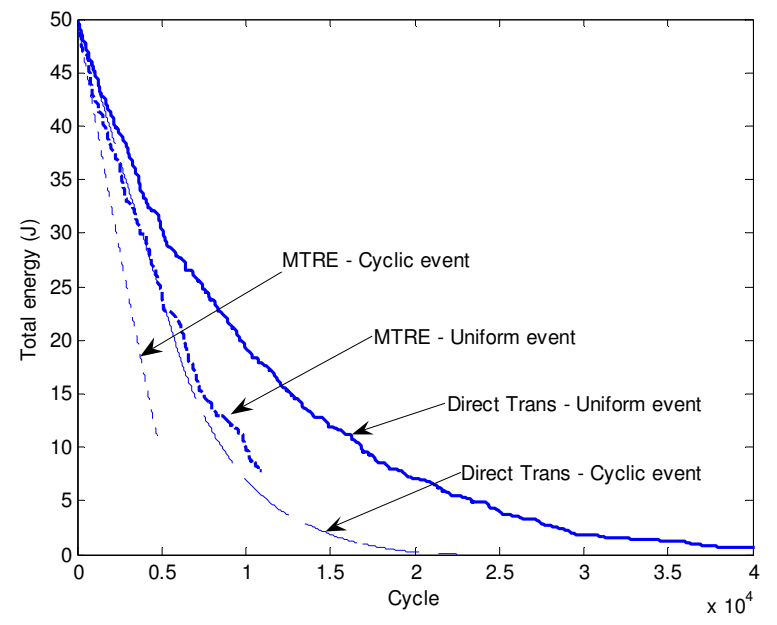

Figure 2. Total adhoc wireless sensor energy dissipated using direct transmission and MTRE with cyclic or uniform events.

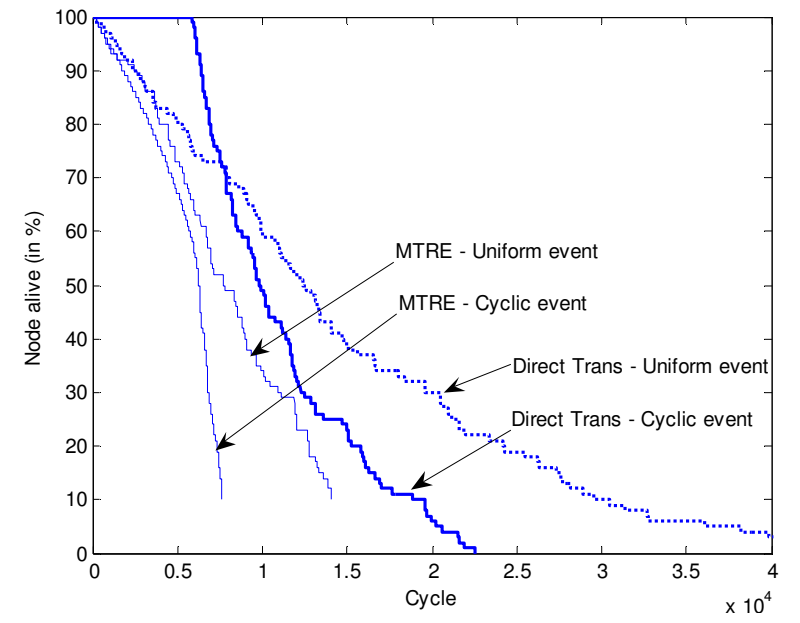

Figure 3. Adhoc wireless sensor network lifetime using direct transmission and MTRE with cyclic or uniform event.

\section{The Static Cluster}

Five relays are randomly chosen among the $N$ nodes over $S$. So, the five clusters of nodes are built around the five relays. The name of the relays and the size of the clusters do not change during the simulation, except if a node is dead in the current cluster. All nodes in the current cluster send their messages to the relay. The relay merges all data and sends to the SN. Hundreds of different topologies of networks are simulated and we average the results (table II). The simulation over one network topology is stoped when the five relays are dead. It is normal, because as the relay is fixed all data go through the relay. And if the relay is dead, no data from nodes could be sent to the SN.

In table II, the first column indicates the number of the network topology. The next five columns indicate the number of nodes in the cluster at the beginning of the life of network. The next five columns indicate the lifetime of each cluster in cycle. And finally, the last column shows the total energy dissipated in the network.

With an onset energy at 50J uniformly distributed over the 100 nodes, only $6 \%$ of energy is dissipated in the network when the five relays are dead. If the number of nodes is distributed equally in each cluster, the lifetime of each cluster will be homogeneous. However, the lifetime of this network will be weaker than the lifetime of network with a disproportionate size of cluster. To conclude, if the same node relay is used for each cluster, the relay works hard and dies quickly.

\section{A variant of $L E A C H$}

The Low Energy Adaptive Clustering Hierarchy (LEACH) algorithm [2] is simulated. At each cycle, the five relays are randomly chosen among the $\mathrm{N}$ nodes. So, the five clusters of nodes are rebuilt around the five relays at each cycle. The simulation is stopped when $50 \%$ of the nodes are dead. The performances of the LEACH are shown in table I. As the size of the cluster of nodes and the relay node change at each cycle, it is impossible to display the same result as table II. 


\section{E. The SOORLECA protocol}

Five relays are randomly chosen in the network. So, the five clusters of nodes are built around the five relays (initial phase). The relay node changes within the cluster all the cycle following the ACMET criterion. The simulation is stopped when $50 \%$ of the nodes are dead.

In table III, the first column indicates the number of the network topology. The next five columns indicate the number of nodes in the cluster at the beginning of the life of the network. The five next columns indicate the lifetime of each cluster in cycle. And finally, the last column shows the total energy dissipated in the network. Also, for a number of network topology the first row gives the number of nodes in each cluster at the beginning life of the network. The following second row shows the nodes still alive.

From the table III, when the number of nodes per cluster is balanced, the lifetime of each cluster is moderately the same. So, the lifetime of the network is grandly improved. But, if the number of nodes per cluster is unbalanced, the lifetime of each cluster is slightly unbalanced. But, if we compare to the lifetime of each cluster for the static cluster algorithm, we show that SOORLECA improves the mean lifetime per cluster. Finally, the lifetime of the network with the SOORLECA is more efficient than the static cluster algorithm.

In table I, a synthesis performances of each algorithm is shown. By comparing the SOORLECA protocol to the others, it is shown that the lifetime of the network is larger than the other algorithms with a mean dissipated energy of $62 \%$. That is to say that the $50 \%$ of nodes have spent the half of the initial energy network. One could think that the $16 \%$ remaining energy comes from the initial phase. So the dissipated energy is smaller than the other algorithms. It is about $31.33 \mathrm{~J}$.

To conclude, the lifetime of the WSN with SOORLECA ACMET 1 protocol is 2 times higher than the LEACH, 6 times higher than Static Cluster, 3.8 times higher than the MTRE and 2.5 times higher than the direct transmission. Besides, the SOORLECA - ACMET 2 dissipates a little more energy than the ACMET1, but it is less efficient than the LEACH for the lifetime of the WSN.

TABLE I. SYNTHESIS OF THE PERFORMANCES OF EACH ALGORITHM.

\begin{tabular}{|l|c|c|}
\hline Algorithms & $\begin{array}{c}\text { Dissipated energy } \\
(\mathbf{J}) \text { and (\%) }\end{array}$ & $\begin{array}{c}\text { Mean lifetime of the } \\
\text { WSN (cycle) }\end{array}$ \\
\hline $\begin{array}{l}\text { Direct transmission } \\
\text { Cyclic event }\end{array}$ & $42.66-85.32 \%$ & 9830 \\
\hline $\begin{array}{l}\text { Direct transmission } \\
\text { Uniform event }\end{array}$ & $34.55-69.1 \%$ & 12410 \\
\hline $\begin{array}{l}\text { MTRE } \\
\text { Cyclic event }\end{array}$ & $41.77-83.55 \%$ & 6245 \\
\hline $\begin{array}{l}\text { MTRE } \\
\text { Uniform event }\end{array}$ & $35.76-71.52 \%$ & 7693 \\
\hline Static Cluster & $2.83-6 \%$ & 4775 \\
\hline LEACH variant & $41,36-82.72 \%$ & 14900 \\
\hline SOORLECA - ACMET 1 & $31.33-62.66 \%$ & 29721 \\
\hline SOORLECA - ACMET 2 & $35.42-70.83 \%$ & 7804 \\
\hline
\end{tabular}

\section{CONCLUSIONS AND FUTUR WORKS}

In this paper, we describe the SOORLECA protocol with the ACMET criteria based on the mean energy level in the cluster. SOORLECA is a clustering based routing protocol that minimizes global energy usage by distributing the load to all the nodes in the network and by distributing the load of the relay work to all the nodes in a cluster at different points in time.

The choice of the energy criterion is significant. A linear choice of potential relay is better than a random in regarding their energy level following a mean energy threshold.

The SOORLECA is completely distributed. It requires no control information from the sink node or from the relays. Also, the nodes do not require knowledge of the global network. But, they only require a local knowledge onto the current cluster. This knowledge is the future relay node for the next cycle of communication.

With an initial uniform distribution of the energy over the network, our simulations show that:

- SOORLECA balances the lifetime of nodes in each cluster even if the number of nodes on the cluster is unbalanced.

- SOORLECA increases significantly the lifetime of the network, 2 times more than the LEACH.

- SOORLECA spends less energy than the MTRE and the LEACH. Only, $61 \%$ of the energy of the network is dissipated when $50 \%$ of nodes are dead.

Also, it appears that a trade-off has to be done with the topology of the network, the energy dissipation by node and also the number of nodes in the cluster.

To confirm our assumptions performances on SOORLECA, we are going to implement the protocol with the OMNET++ platform simulation [15],[16] and MICA2 wireless measurement system [17].

Based on our MATLAB simulations describe above, we are confident that SOORLECA will outperform conventional communication protocols in terms of energy dissipation and lifetime of the network.

\section{REFERENCES}

[1] C. Intanagonwiwat, R. Govindan and D. Estrin, "Directed diffusion: a scalable and robust communication paradigm for sensor networks", in Proceedings of ACM MOBICOM'00, Boston, MA, 2000.

[2] W. Heinzelman, A. Chandrakasan, and H. Balakrishnan, "EnergyEfficient Communication Protocol for Wireless Microsensor Networks", In Proceedings of the Hawaii Conference on System Sciences, Jan. 2000.

[3] F. Ye, H. Luo, J. Cheng, S. Lu and L. Zhang, "A two tier data dissemination model for large-scale wireless sensor networks", in Proceedings of ACM MOBICOM'02, Atlanta, GA, September 2002.

[4] W. Heinzelman, J. Kulik and H. Balakrishnan, "Adaptive Protocols for Information Dissemination in ", Wireless Sensor Networks ACM International Conference on Mobile Computing and Networking (MOBICOM'99), 1999.

[5] S. Lindsey and C. S. Raghavendra, "PEGASIS: Power efficient gathering in sensor information systems", in Proceedings of the IEEE Aerospace Conference, Vol. 3, pp. 1125-1130, Montana, USA, March 2002. 
[6] D. Estrin and all, "Next century challenges: Scalable coordination in sensor networks", in Proceedings of the $5^{\text {th }}$ annual ACM/IEEE international conference on Mobile Computing and Networking (MobiCom '99), pp. 263-270, Seattle, USA, August 1999.

[7] T. He and all, "Range-free localization schemes for large scale sensor networks", in Proceedings of the $9^{\text {th }}$ annual ACM/IEEE international conference on Mobile Computing and Networking (MobiCom '03), pp. 81-95, San Diego, USA, September 2003.

[8] T. S. Rappaport, Wireless Communications, Prentice-Hall, 1996.

[9] J. Proakis, "Digital communications", Mc Graw Hill, 5th edition, ISBN13 : 978-0-07-126378-8, 2008.

[10] R. Steele, "Mobile Radio Communications", Pentech Press, London, 1992.

[11] D. Hall, "Mathematical Techniques in Multisensor Data Fusion", Artech House, Boston, MA, 1992.
[12] L. Klein, "Sensor and Data Fusion Concepts and Applications", SPIE Optical Eng Press, WA, 1993.

[13] P. Fan and M. Darnell, "Sequence design for communications applications", Research studies press LTD, John Wiley\&sons inc., ISBN 086380-201, 1996.

[14] N. M. Murad, "A Self Organization One hop Relay Low Energy Clustering Adaptation protocol for an adhoc wireless sensor network", Sixth Annual IEEE Communications Society Conference on Sensor, Mesh and Ad Hoc Communications and Networks, SECON 2009, submitted to review, June $22-26^{\text {th }}$, Rome, Italy.

[15] Omnet++ plateform simulation, http://www.omnetpp.org/

[16] J. Hill and all, "System architecture directions for networked sensor", in Proceddings of the $9^{\text {th }}$ Internationnal Conference on Architectural Support for Programming languages and Operating Systems, November 2000.

[17] Crossbow Technology Inc. , http://www.crossbow.com

TABLE II. PERFORMANCE OF THE STATIC CLUSTER

\begin{tabular}{|c|c|c|c|c|c|c|c|c|c|c|c|}
\hline $\begin{array}{c}\mathbf{n}^{\circ} \\
\text { network }\end{array}$ & $\begin{array}{c}\text { Cluster } \\
1\end{array}$ & $\begin{array}{c}\text { Cluster } \\
2\end{array}$ & $\begin{array}{c}\text { Cluster } \\
3\end{array}$ & $\begin{array}{c}\text { Cluster } \\
4\end{array}$ & $\begin{array}{c}\text { Cluster } \\
5\end{array}$ & \begin{tabular}{|c|} 
Time dead \\
C1
\end{tabular} & $\begin{array}{c}\text { Time dead } \\
\text { C2 }\end{array}$ & $\begin{array}{c}\text { Time dead } \\
\text { C3 }\end{array}$ & \begin{tabular}{|c|} 
Time dead \\
C4
\end{tabular} & $\begin{array}{c}\text { Time dead } \\
\text { C5 }\end{array}$ & $\begin{array}{l}\text { Dissipated } \\
\text { energy }(\mathrm{J})\end{array}$ \\
\hline 1 & 4 & 4 & 27 & 10 & 50 & 229 & 241 & 7 & \begin{tabular}{|l|}
43 \\
\end{tabular} & 2 & 2.90 \\
\hline 2 & 20 & 39 & 11 & 19 & 6 & 12 & 4 & 36 & 13 & 115 & 2.81 \\
\hline 3 & 28 & 15 & 16 & 16 & 20 & 7 & 20 & 18 & 18 & 12 & 2.73 \\
\hline 4 & 16 & 10 & 34 & 30 & 5 & 17 & 44 & 4 & 5 & 154 & 2.92 \\
\hline$\ldots$ & $\ldots$ & $\ldots$ & $\ldots$ & $\ldots$ & $\ldots$ & $\ldots$ & $\ldots$ & $\ldots$ & $\ldots$ & $\ldots$ & $\ldots$ \\
\hline 17 & 20 & 14 & 24 & 16 & 21 & 11 & 23 & 8 & 18 & 11 & 2.79 \\
\hline 18 & 13 & 33 & 22 & 17 & 10 & 26 & 5 & 10 & 16 & 42 & 2.82 \\
\hline 19 & 9 & 18 & 23 & 11 & 34 & 52 & 14 & 9 & 35 & 4 & 2.80 \\
\hline 20 & 9 & 11 & 2 & 8 & 65 & 53 & 34 & 802 & 65 & 2 & 2.97 \\
\hline$\ldots$ & $\ldots$ & $\ldots$ & $\ldots$ & $\ldots$ & $\ldots$ & $\ldots$ & $\ldots$ & $\ldots$ & $\ldots$ & $\ldots$ & $\ldots$ \\
\hline 47 & 24 & 19 & 25 & 15 & 12 & 9 & 13 & 8 & 20 & 31 & 2.77 \\
\hline$\ldots$ & $\ldots$ & $\ldots$ & $\ldots$ & $\ldots$ & $\ldots$ & $\ldots$ & $\ldots$ & $\ldots$ & $\ldots$ & $\ldots$ & $\ldots$ \\
\hline 89 & 20 & 28 & 7 & 21 & 19 & 12 & 6 & 75 & 11 & 13 & 2.80 \\
\hline 90 & 26 & 21 & 8 & 26 & 14 & 7 & 11 & 61 & 7 & 23 & 2.81 \\
\hline$\ldots$ & $\ldots$ & $\ldots$ & $\ldots$ & $\ldots$ & $\ldots$ & $\ldots$ & $\ldots$ & $\ldots$ & $\ldots$ & $\ldots$ & $\ldots$ \\
\hline 98 & 53 & 30 & 6 & 4 & 2 & 2 & 5 & 116 & 238 & 827 & 3.00 \\
\hline 99 & 13 & 13 & 18 & 37 & 14 & 26 & 26 & 14 & 4 & 22 & 2.81 \\
\hline 100 & 5 & 9 & 45 & 14 & 22 & 161 & 47 & 3 & 23 & 10 & 2.87 \\
\hline Mean & 18.92 & 21.72 & 18.11 & 18.10 & 18.15 & 42 & 44 & 55 & 35 & 62 & 2.83 \\
\hline
\end{tabular}

TABLE III. PERFORMANCE OF THE SOORLECA PROTOCOL

\begin{tabular}{|c|c|c|c|c|c|c|c|c|c|c|c|}
\hline \begin{tabular}{|c|}
$\mathbf{n}^{\circ}$ \\
network
\end{tabular} & $\begin{array}{c}\text { Cluster } \\
1\end{array}$ & $\begin{array}{c}\text { Cluster } \\
2\end{array}$ & $\begin{array}{c}\text { Cluster } \\
3\end{array}$ & $\begin{array}{c}\text { Cluster } \\
4\end{array}$ & $\begin{array}{c}\text { Cluster } \\
5\end{array}$ & $\begin{array}{c}\text { Time dead } \\
\text { C1 }\end{array}$ & $\begin{array}{c}\text { Time dead } \\
\text { C2 }\end{array}$ & $\begin{array}{c}\text { Time dead } \\
\text { C3 }\end{array}$ & \begin{tabular}{|c|} 
Time dead \\
C4
\end{tabular} & $\begin{array}{c}\text { Time dead } \\
\text { C5 }\end{array}$ & $\begin{array}{l}\text { Dissipated } \\
\text { energy }(\mathbf{J})\end{array}$ \\
\hline 1 & 25 & 28 & \begin{tabular}{l|l}
17 & \\
\end{tabular} & 20 & 10 & - & - & - & - & - & - \\
\hline 1 & 12 & 13 & 8 & 9 & 4 & 205 & 201 & 280 & 286 & 635 & 29.86 \\
\hline 2 & 16 & 19 & 23 & 31 & 11 & - & - & - & - & - & - \\
\hline 2 & 7 & 9 & 11 & 15 & 5 & 336 & 262 & 227 & 169 & 463 & 29.50 \\
\hline$\ldots$ & $\ldots$ & $\ldots$ & $\ldots$ & $\ldots$ & $\ldots$ & $\ldots$ & $\ldots$ & $\ldots$ & $\ldots$ & $\ldots$ & $\ldots$ \\
\hline 50 & 62 & 32 & 15 & 13 & 29 & - & - & - & - & - & - \\
\hline 50 & 30 & 15 & 7 & 6 & 14 & 52 & 160 & 315 & 338 & 167 & 31.21 \\
\hline 51 & 21 & 31 & 38 & 23 & 9 & - & - & - & - & - & - \\
\hline 51 & 10 & 15 & 18 & 11 & 4 & 241 & 164 & 133 & 89 & 583 & 30.48 \\
\hline$\ldots$ & $\ldots$ & $\ldots$ & $\ldots$ & $\ldots$ & $\ldots$ & $\ldots$ & $\ldots$ & $\ldots$ & $\ldots$ & $\ldots$ & $\ldots$ \\
\hline 97 & 26 & 22 & 9 & 98 & 14 & - & - & - & - & - & - \\
\hline 97 & 12 & 10 & 4 & 47 & 6 & 192 & 235 & 525 & 61 & 424 & 35.38 \\
\hline 98 & 32 & 99 & 11 & 3 & 31 & - & - & - & - & - & - \\
\hline 98 & 15 & 49 & 5 & 1 & 15 & 157 & 62 & 443 & 1967 & 98 & 31.68 \\
\hline 99 & 27 & 38 & 30 & 30 & 80 & - & - & - & - & - & - \\
\hline 99 & 13 & 17 & 13 & 14 & 39 & 189 & 57 & 63 & 150 & 52 & 33.00 \\
\hline 100 & 26 & 15 & 29 & 24 & 68 & - & - & - & - & - & - \\
\hline 100 & 12 & 7 & 14 & 11 & 33 & 113 & 290 & 165 & 207 & 59 & 32.33 \\
\hline & & & & & Mean & 276 & 265 & 303 & 343 & 297 & 31.33 \\
\hline
\end{tabular}

